Revue internationale P.M.E.

Économie et gestion de la petite et moyenne entreprise

\title{
Pour une lecture des problèmes complexes en PME: approche conceptuelle et expérimentation
}

\section{Christophe Schmitt, Pierre-André Julien et Richard Lachance}

Volume 15, numéro 2, 2002

URI : https://id.erudit.org/iderudit/1008806ar

DOI : https://doi.org/10.7202/1008806ar

Aller au sommaire du numéro

Éditeur(s)

Presses de l’Université du Québec

ISSN

0776-5436 (imprimé)

1918-9699 (numérique)

Découvrir la revue

Citer cet article

Schmitt, C., Julien, P.-A. \& Lachance, R. (2002). Pour une lecture des problèmes complexes en PME: approche conceptuelle et expérimentation. Revue internationale P.M.E., 15(2), 35-62. https://doi.org/10.7202/1008806ar

\section{Résumé de l'article}

Les problèmes complexes affectent toutes les formes d'organisation, y compris les PME, et incitent leurs dirigeants à rechercher des outils relativement simples pour faire face à la complexité et mieux asseoir leur stratégie. Dans cet article, à l'aide d'une démarche constructiviste et à la suite d'une analyse de la complexité, nous présentons un tel outil grâce auquel nous pouvons mettre en relation les variables qui caractérisent une situation et les hiérarchiser dans un modèle de représentation comportant quatre catégories, soit leur influence, leurs enjeux, leur dépendance et leur autonomie. Cette hiérarchisation permet aux décideurs de s'attaquer rapidement aux variables qui affectent le plus les autres variables ou qui conditionnent le système par l'importance de leur influence. Nous montrons, de plus, comment peut fonctionner cet outil en l'appliquant à un cas réel d'entreprise à travers un audit complexe fournissant un plan d'amélioration continue. 


\title{
Pour une lecture des problèmes complexes en PME : approche conceptuelle et expérimentation
}

\author{
Christophe SCHMITT \\ Université de Metz \\ Pierre-André JULIEN \\ Richard LACHANCE ${ }^{1}$ \\ Institut de recherche sur les PME, \\ Université du Québec à Trois-Rivières
}

MOTS CLÉS

Problèmes complexes - Stratégie des PME
Situations paradoxales - Paradigme constructiviste
Modèle topographique de représentation
Caractérisation des entreprises - Plan de développement continu

\section{RÉSUMÉ}

Les problèmes complexes affectent toutes les formes d'organisation, y compris les $P M E$, et incitent leurs dirigeants à rechercher des outils relativement simples pour faire face à la complexité et mieux asseoir leur stratégie. Dans cet article, à l'aide d'une démarche constructiviste et à la suite d'une analyse de la complexité, nous présentons un tel outil grâce auquel nous pouvons mettre en relation les variables

1. Les auteurs tiennent à remercier Steeve Kearney et Richard Lacoursière pour leur travail sur le terrain ainsi que les trois lecteurs pour leurs remarques constructives.

\section{LES AUTEURS}

CHRISTOPHE SCHMITT est maître de conférences à l'Université de Metz (IUT GEA) et membre du CEREMO. Il est actuellement chargé de mission et responsable du DESS «Entrepreneuriat et Développement des PME » (ESM-IAE de Metz). Ces travaux de recherche portent sur la dynamique des organisations, et notamment des PME, et sur l'ingénierie des pratiques entrepreneuriales. Adresse: Université de Metz, CEREMO, 3, place Édouard-Branly, 57070 Metz. Courriel : <schmitt@esm.univ-metz.fr>. 
qui caractérisent une situation et les hiérarchiser dans un modèle de représentation comportant quatre catégories, soit leur influence, leurs enjeux, leur dépendance et leur autonomie. Cette hiérarchisation permet aux décideurs de s'attaquer rapidement aux variables qui affectent le plus les autres variables ou qui conditionnent le système par l'importance de leur influence. Nous montrons, de plus, comment peut fonctionner cet outil en l'appliquant à un cas réel d'entreprise à travers un audit complexe fournissant un plan d'amélioration continue.

\begin{abstract}
Complex problems affect every type of organizations, including small and mediumsized enterprises (SMEs). Managers thus search for relatively simple tools to help them solve these problems and sustain their strategy. In this paper, using a constructivist view and an analysis of complexity, we present such a tool. Its function is to represent the relationships between the various variables that characterize a problem situation and to prioritize them, using a representation model that includes four categories, namely the influence level of the variables, their strategic importance, their level of dependency and their level of autonomy. This prioritization allows decision-makers to focus on the variables that most affect others or that condition the system by the importance of their influence. We also show how this tool can function by applying it to an actual case, following a complex audit that provides a plan for continuous improvement.
\end{abstract}

\title{
RESUMEN
}

Los problemas complejos afectan todas las formas de organización, comprendidas las pequeñas y medianas empresas (PyMEs), y confrontan asi a sus dirigentes a buscar instrumentos relativemente simples que puedan ayudarles a hacer frente para asentar mejor su estrategia. Es este articulo, dentro de una gestión cosntructivista y después de un análisis de la complejidad, nosotros presentamos tal instrumento, permitiendo poner en relación las diferentes variaciones que

Pierre-André Julien détient un doctorat en sciences économiques de l'Université catholique de Louvain et un doctorat honoris causa de l'École nationale polytechnique de Lorraine. II est titulaire de la Chaire Bombardier Produits récréatifs en gestion du changement technologique dans les PME et directeur de la Revue. Présentement, il travaille avant tout sur la gestion de l'information dans les PME et dans les réseaux d'entreprises. II a publié à ce jour près de 80 articles dans des revues internationales et 19 ouvrages. Adresse: Institut de recherche sur les PME, Université du Québec à Trois-Rivières, 3351, boulevard des Forges, C.P.500, Trois-Rivières, Québec, Canada, G9A 5H7. Courriel : <pierre-andre_julien@uqtr.ca>.

RICHARD LACHANCE est professionnel de recherche à l'Université du Québec à TroisRivières depuis 1989. Il détient une maîtrise en économie et gestion des PME (MSc). Depuis 1994, il travaille à la Chaire Bombardier Produits récréatifs (chaire en gestion du changement technologique dans les PME). II participe aux diagnostics organisationnels des PME membres. Depuis quelques années, il s'intéresse plus particulièrement à l'analyse des réseaux sociaux dans un contexte d'affaires : réseaux de PME en région ou articulées autour d'une entreprise pivot (relations donneurs d'ordres et sous-traitants). Adresse : Institut de recherche sur les PME, Université du Québec à Trois-Rivières, 3351, boulevard des Forges, C.P. 500, Trois-Rivières, Québec, Canada, G9A 5H7. Courriel : <richard_lachance@uqtr.ca>.

Revue internationale P.M.E., vol. $15, \mathrm{n}^{\circ}$ 2, 2002 
caracterizan una situación y de las jerarquizar en un modelo de representación comportando cuatro categorias según su nivel de influencia, de riesgos, de dependencia y de autonomia. Esta jerarquización, permite a los que deciden, atacarse rápidamente a las variaciones que más afectan a las otras variaciones $o$ que condicionan el sistema por la importancia de su influencia. Nosotros además enseñamos, como puede funcionar este instrumento aplicándolo a un caso real de empresa después de efectuar una auditoria compleja, ofreciéndole un plan de mejoramiento a término medio.

\section{ZUSAMMENFASSUNG}

Die komplexen Probleme verlangen nach allen Formen von Organisationen, inbegriffen den $K M U$, und führen ihre Unternehmer dazu, nach einfachen Instrumenten suchen um der Komplexität zu entgegnen und ihre Strategie besser zu stabilisieren. In diesem Artikel wird mit der Hilfe einer aufbauenden Vorgehensweise und als Folge einer Analyse der Komplexität, ein solches Instrument vorgestellt. Dieses Instrument erlaubt, die für eine Situation charakteristischen Variablen einzubeziehen, und sie in einem Verhaltens-Modell zu ordnen. Das Modell enthält vier Kategorien, das Einflussniveau, der Einsatz, die Abhängigkeit und die Autonomie der Variablen. Diese hierarchische Ordnung erlaubt den Entscheidungsträgern diejenigen Variablen schnell in Angriff zu nehmen, welche anderen Variablen am meisten beeinträchtigen oder die das System durch die Bedeutung ihres Einflusses bestimmen. Wir zeigen im weiteren, wie dieses Instrument funktioniert, bei einer Unternehmung unmittelbar nach einem komplexen Audit und nach einem ständigen Verbesserungsplan.

\section{Introduction}

Ce qui distingue dès l'abord le plus mauvais architecte de l'abeille la plus experte, c'est qu'il a construit la cellule dans sa tête avant de la construire dans la ruche.

Karl MARX

Les PME, comme toutes les organisations, font régulièrement face à des problèmes qui se caractérisent par leur complexité2 à savoir des situations problématiques dont les acteurs ne se satisfont pas et qu'ils souhaitent modifier. Si des recherches orientées vers la compréhension de ces phénomènes voient le jour depuis quelques années (Chanal, Lesca et Martinet, 1997 ; Girin, 1990 ; Lukka, 1998 ; Kaplan, 1998), à notre connaissance, peu de travaux dans ce domaine s'intéressent à la PME. Il s'agit donc ici de montrer la spécificité de la PME au regard de ce type de problèmes et de proposer, à côté des outils existants telles les cartes cognitives (Cossette, 1994 ;

2. Nous définissons la complexité comme la caractéristique d'un système dans lequel circulent des flux nombreux, diversifiés, multidirectionnels, riches, qui permettent au système de prendre des états variés.

Revue internationale P.M.E., vol. $15, \mathrm{n}^{\circ}$ 2, 2002 
Verstraete, 1997), une démarche et d'autres outils pouvant servir à rendre intelligible la complexité des situations à gérer en PME. Ainsi, nous visons à favoriser l'action des dirigeants dans un environnement considéré comme complexe.

L'originalité de notre contribution repose sur deux points :

- une lecture de la PME en termes de complexité. Nous mettons en évidence les difficultés éprouvées par les dirigeants des PME dans le traitement des problèmes complexes. En effet, les pratiques observées, convergentes mais limitées, font émerger des situations paradoxales (Schmitt, 1999);

- une proposition méthodologique pour aborder la complexité en PME. Nous présentons le cadre épistémologique approprié pour lever le paradoxe et les implications méthodologiques que cela entraine (Le Moigne, 1990).

Cet article est le fruit d'une collaboration entre des équipes de recherche lorraine et québécoise ${ }^{3}$. Il correspond à l'expérience acquise au sein de ces équipes, tant dans le domaine de la gestion que de l'ingénierie, à travers des travaux de thèse, de contrats de recherche et d'applications sur le terrain.

Ainsi, nous avons articulé cet article en quatre parties autour de la problématique de la compréhension et de l'appréhension des problèmes complexes en PME. Nous présentons, dans un premier point, la relation entre PME et complexité autour de trois éléments : la présentation du contexte de la PME, la spécificité de la PME et l'émergence de situations paradoxales. Dans un deuxième point, nous abordons le cadre d'une recherche adaptée aux problèmes complexes en contexte de PME. L'objectif de cette section est de considérer la complexité comme un construit. Cela a pour conséquence non seulement de considérer les problèmes avant tout comme des problèmes de représentation, mais aussi de faire la distinction entre problèmes complexes et non complexes et, donc, d'introduire des modes de résolution différents. Dans un troisième point, afin d'illustrer nos propos, nous relatons une expérience menée dans une PME et présentons les éléments d'instrumentation nécessaires à la mise en œuvre de ce type de projet. Enfin, tout en soulignant le caractère inachevé de cette entreprise, le dernier point est l'objet d'une discussion sur les outils utilisés ainsi que d'une présentation d'un cadre plus général pour ce type de recherche en PME.

3. Il s'agit d'un projet piloté par l'Institut lorrain des sciences du travail, de l'emploi et de la formation, regroupant les laboratoires du CEREMO (Metz), du GREFIGE et du LRGSI (Nancy) et, au Québec, de la Chaire Bombardier en gestion du changement technologique dans les PME de l'Institut de recherche sur les PME à l'UQTR.

Revue internationale P.M.E., vol. 15, $\mathrm{n}^{\circ}$ 2, 2002 


\section{La problématique de la complexité en PME}

\subsection{Problèmes complexes et PME : présentation du contexte}

La recherche présentée dans cet article a pour point de départ l'hypothèse communément admise dans la pratique que nombre de problèmes que sont appelées à résoudre les PME sont des problèmes complexes, même si les dirigeants n'en sont pas forcément conscients. Ces problèmes, dont la documentation scientifique sur la PME se fait souvent l'écho, sont nombreux et variés :

- développer un nouveau produit (Damanpour, 1996; Lopez-Monsalvo, 1998);

- mettre en place des éléments de gestion de production (qualité, juste à temps, etc.; Messeghem et Varraut, 1998 ; Gélinas et al., 1996);

- s'adapter à son environnement (comme exemple, répondre à des commandes supplémentaires ou à des nouvelles commandes, apparition de nouveaux concurrents, etc.; Delobel, 1998 ; Schmitt et Bayad, 2000);

- gérer le personnel (absence, embauche, rotation ou encore mise en place d'équipes supplémentaires de production ; Fabi, 1997 ; Bayad et Paradas, 1998 ; Tarondeau, 1998 ; Jacob, Julien et Raymond, 1996).

Quelle que soit la nature de ces problèmes - évolution interne ou externe, modifications législatives ${ }^{4}$, pilotage de projets innovants, introduction de nouvelles technologies ou mise en place d'une démarche de changement -, ils ont en commun d'être « dés-organisants 5 » pour l'entreprise. Ces problèmes étant complexes, ils ont un impact direct sur l'adaptabilité des structures, des buts et des moyens liés à leur processus de création de valeur (Gervais, 1991) et pour conséquence indirecte de remettre en question la stratégie de l'entreprise par rapport à son environnement (Martinet, 1993). Ce constat reflète les problèmes quotidiens qui se posent aux dirigeants de PME : créer de la valeur dans un contexte considéré comme complexe (Marchesnay et Carrier, 2002).

\subsection{Problèmes complexes et PME : la spécificité des PME}

Les difficultés pour aborder ce type de problèmes ne doivent pas être considérées comme une caractéristique de la PME, car elle ne vit pas forcément plus de problèmes que d'autres types d'organisation (Blackstone et Cox, 1985). La complexité

4. Exemples : la loi sur les 35 heures hebdomadaires de travail en France, la loi sur l'équité salariale qui vient d'entrer en vigueur au Québec.

5. Pour insister sur l'aspect désorganisant de la complexité que nous présentons dans cet article, nous avons choisi de l'écrire comme le résultat de la contraction des notions de désordre et d'organisation.

Revue internationale P.M.E., vol. 15, nº 2, 2002 
n'est certes pas l'apanage des entreprises de petite et moyenne taille, mais les problèmes qui surgissent sont le reflet de leur spécificité. À la lumière de la définition de la PME (Julien, 2002), il est possible de relever les sources potentielles de difficultés pour traiter des problèmes complexes. Ainsi, nous pouvons dégager trois axes principaux :

- la centralisation de la gestion. Les actions entreprises sont rattachées à la volonté du dirigeant, c'est-à-dire sa vision stratégique, sa philosophie de gestion et ses pratiques managériales (Bayad et Nebenhaus, 1998). Généralement, les solutions retenues reflètent les représentations ${ }^{6} \mathrm{du}$ dirigeant, alors que la complexité des problèmes qui se posent nécessite de confronter les représentations des acteurs de l'organisation. Le problème étant élaboré le plus souvent par une seule personne, il souffre d'une difficulté de conception appauvrissant les solutions potentielles apportées aux problèmes. De plus, la complexité affecte, par ses interactions, différentes dimensions de l'entreprise que le dirigeant doit considérer comme des dimensions organisationnelles et opérationnelles. En résumé, le dirigeant de PME est typiquement une personne qui évolue dans la complexité ;

- une stratégie intuitive ou peu formalisée. Pour résoudre les problèmes qui se posent, le dirigeant élabore des logiques d'actions à court terme (Marchini, 1995; Torrès, 1999), alors que, comme le souligne Morin (1990), « la complexité appelle la stratégie». Nous avons pu constater que les dirigeants parlent à la fois de la nécessité de regarder plus loin (par exemple, dans le cadre d'une vision; Filion, 1991) et d'avouer que c'est exactement le contraire qui se passe dans la réalité parce qu'ils doivent toujours s'occuper des problèmes les plus urgents. Dans ces conditions, il existe des risques d'effets de type loi de GRESHAM selon laquelle des logiques court-termistes limitent le répertoire de solutions à appliquer à long terme et s'inscrivent dans «une illusion de choix» (Watzlawick, Weakland et Fisch, 1974). Autrement dit, les mauvaises pratiques chassent les bonnes ou d'éventuelles meilleures (Godet, 1994). Dans ces conditions, l'un des objectifs de notre recherche est de libérer le dirigeant de cette vision à court terme pour qu'il puisse consacrer du temps à la construction d'un horizon temporel plus large (Julien et al., 2000);

6. Nous retenons comme définition de la notion de représentation : état de l'image mentale que se fait un sujet ou un groupe d'un objet, d'un concept, d'un contexte, d'un événement, d'un système, d'un comportement, plus généralement. Il s'agit d'une (re)construction circonstancielle du réel faite dans un contexte particulier et à des fins spécifiques, à savoir dans une situation donnée et pour faire face aux exigences de la tâche en cours.

Revue internationale P.M.E., vol. 15, $\mathrm{n}^{\circ}$ 2, 2002 
- l'absence ou la quasi-absence d'outils pour aborder la complexité des situations. La gestion des situations complexes est guidée par l'expérience et le bon sens plutôt que par l'utilisation de techniques spécifiques (Torrès, 1999). En effet, pour régler les problèmes, les responsables de PME ne disposent que de peu d'outils pour percevoir la complexité des situations et encore moins pour se la représenter (Avenier, 1988). De plus, les problèmes sont envisagés pour eux-mêmes et rarement par rapport aux interactions avec l'organisation. Force est de constater que la rationalité limitée des individus ne permet pas de tenir compte de toutes les interactions du problème (Simon, 1991). En d'autres termes, la complexité est abordée par la simplicité, comme si le complexe n'était qu'un niveau de complication supplémentaire.

\subsection{Problèmes complexes et PME : l'émergence de situations paradoxales}

Dans ces conditions, la gestion des problèmes complexes en PME doit être qualifiée de centralisée mais limitée. En effet, elle se traduit souvent par l'émergence d'un paradoxe: les efforts consentis par les dirigeants des PME par rapport à la complexité des problèmes, non seulement ne sont pas efficaces mais, en plus, engendrent d'autres problèmes, comme par exemple une baisse de la qualité des produits, une augmentation des délais de livraison ou, encore, une augmentation des coûts de fabrication. En d'autres termes, «la solution est le problème » (Watzlawick, Weakland et Fisch, 1974).

\section{Exemple de situations paradoxales en PME : le cas de l'entreprise B}

L'entreprise $B$ est une entreprise de près de 50 employés dans le domaine de la confiserie en France. Plusieurs fois dans l'année, le responsable des ventes accepte des commandes de clients alors que la capacité de production est considérée, du point de vue du reste du personnel de l'entreprise et, notamment, du responsable de production, à son maximum. Devant l'afflux de la demande, l'entreprise essaie de trouver des solutions pour honorer ces contrats. Généralement, elle s'organise en adaptant son volume de main-d'œuvre. Soit elle fait appel à l'agence d'intérim avec laquelle elle a l'habitude de travailler, soit elle utilise son propre réseau de recrutement, telles des personnes qu'elle utilise souvent lors de pénuries de main-d'œuvre (absence du personnel pour maladie, congés payés, formation, etc.), soit elle utilise les deux solutions. Mais les résultats sont souvent loin d'atteindre les objectifs fixés. En effet, l'entreprise constate souvent, dans ces situations, une baisse de la qualité et un manque d'homogénéisation des produits, une augmentation du temps du processus de fabrication. Pour résoudre ces problèmes, l'entreprise essaie d'employer les mêmes personnes pour éviter les charges financières et temporelles liées à la formation. En agissant ainsi, elle propose «toujours plus de la même chose» (Watzlawick, 1988) et ne fait que limiter les conséquences du phénomène d'apprentissage lié notamment au mode opératoire.

Revue internationale P.M.E., vol. 15, $\mathrm{n}^{\circ}$ 2, 2002 
Plus généralement, qualifier un problème de complexe, c'est reconnaître « la présence de difficultés rencontrées lors de tentatives passées pour les résoudre et faire le constat d'une insatisfaction face aux résultats obtenus jusqu'à présent » (Landry, 1983). Ainsi, l'existence de paradoxes constitue une expression parmi d'autres de la complexité. Ces différents constats nous amènent à déduire que les difficultés qu'ont à surmonter les PME sont plus liées à la construction des problèmes complexes qu'à la mise en place de solutions. Les problèmes auxquels font face les dirigeants de PME se posent donc en termes d'outils pour appréhender la complexité.

Dans ces conditions, la question centrale abordée dans la suite de l'article est celle-ci : «Quels sont les outils pour aborder la complexité des situations en PME ?» Pour cela, il est nécessaire de présenter, dans un premier temps, le cadre épistémologique retenu puis la démarche et les outils mis en place.

\section{Le paradigme constructiviste : un cadre épistémologique approprié à la complexité}

Un paradoxe n'a d'intérêt que si l'on cherche à en sortir. Alors que pour lever un paradoxe logique, il est nécessaire de connaître les enchaînements de causalité, lever un paradoxe comme celui qu'engendrent les situations présentées précédemment dépend essentiellement de l'appréhension des interactions, c'est-à-dire de l'intelligibilité des problèmes complexes (Barel, 1979).

\subsection{Complexité et système de représentation}

Le recours à la complexité ne doit pas être envisagé comme une «entreprise de démission intellectuelle qui consiste à affirmer que tout est complexe » (Weinberg, 1995), mais comme la possibilité de trouver un mode de gestion approprié au contexte actuel des entreprises. Les pratiques managériales auxquelles on recourt dans les PME pour résoudre des problèmes complexes s'inscrivent dans un cadre de référence ontologique où la réalité est considérée comme une donnée indépendante de l'observateur et antérieure à l'observation (Girod-Séville et Perret, 1999). Or, la complexité doit être envisagée avant tout comme un construit humain (Génelot, 1992). La complexité est donc liée à «l'ensemble des circonstances dans lesquelles une personne se trouve » (Schlanger, 1990), c'est-à-dire à une situation. Ainsi, l'évidence ontologique de la complexité se trouve renversée au profit d'une approche axiologique, qui engage les valeurs des personnes concernées et le sens donné aux situations rencontrées. Ce changement de paradigme agit comme «un mécanisme perceptif et cognitif qui transforme la réalité en représentation » (Le Moigne, 1990) et il n'est pas sans incidence : il impose des concepts, des méthodes et des outils différents. En effet, l'objectif n'est plus d'accumuler des connaissances 
substantielles, c'est-à-dire portant sur le contenu et engendrant des outils à utiliser dans une situation déterminée, mais de produire des connaissances sur les processus (Julien et Marchesnay, 1992), plus aptes à modifier la manière d'agir.

Plus généralement, dans un objectif de création de valeur, les situations paradoxales rencontrées par le personnel des PME sont donc liées à leur propre système de représentation. Or, comme le souligne Nicot (1997), « les membres de l'organisation ne le perçoivent pas toujours en tant que tel et ils n'ont plus conscience de cette activité représentative ». Le devenir de l'organisation passe par sa capacité à remettre en question son système de représentation, c'est-à-dire à réaliser une accommodation au sens de Piaget (1973).

\subsection{Problèmes complexes et problèmes «non complexes »}

Dans cette perspective, les solutions que requiert la complexité ne se créent pas de façon mimétique en se référant à des problèmes non complexes. En effet, alors que la résolution de problèmes non complexes présuppose l'existence d'éléments minimes et insécables, la résolution de problèmes complexes nécessite de supposer « une continuité holiste» (Bergandi, 1998), favorisant l'intelligibilité de la complexité. Il est donc possible de dégager deux cadres d'action suivant la nature des problèmes posés.

Figure 1

Le positionnement des différents types de problème dans une perspective de création de valeur

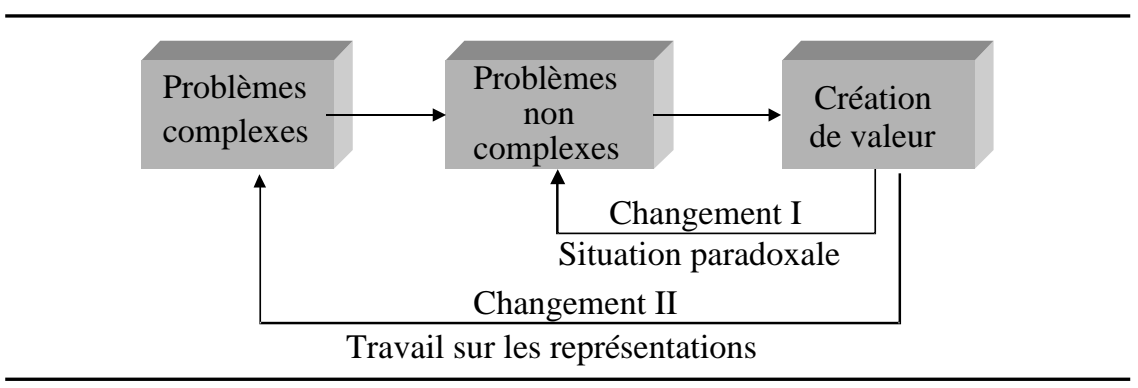

- les changements de type $\mathrm{I}^{7}$ respectent les cadres préétablis. Ils se font à l'intérieur du système sans modifier ce dernier. Il s'agit d'une adaptation, c'est-à-dire d'un ajustement à des conditions que l'entreprise estime ne

7. Watzlawick, Weakland et Fisch (1974) relèvent deux types de changement : le premier ramène l'équilibre antérieur (changement I) et le second favorise les capacités d'apprentissage et d'innovation (changement II).

Revue internationale P.M.E., vol. 15, nº 2, 2002 
pas pouvoir contrôler. L'objectif consiste à adapter les pratiques de court terme (en usage), ou routines, sans les remettre en cause. Ces changements sont appropriés aux problèmes non complexes;

- les changements de type II, à partir desquels l'entreprise peut estimer nécessaire de modifier la structure même de ses actions et de revoir son système de représentation. Ces changements représentent « une remise en question des normes et valeurs organisationnelles et une reconstruction du cadre général de référence » (Probst et Büchel, 1995). On parlera alors de construction ${ }^{8}$, laissant entendre que les situations ne sont pas données et que les comportements, quoique contraints, ne sont pas déterminés (Koenig, 1996). Ces changements sont des réponses appropriées à la complexité des problèmes à résoudre.

Dans ce cas, la mise en place de solutions inadéquates favorise l'émergence de situations paradoxales en PME. En effet, ces solutions relèvent d'un changement de type I, alors que la complexité des problèmes aurait nécessité des changements de type II.

\subsection{La nécessité de modéliser}

Aborder les problèmes complexes et ainsi éviter le risque d'émergence de situations paradoxales exige un travail de compréhension basé sur une modélisation originale mais efficace, favorisant l'intelligence de la situation (Michel, 1997). Les outils qui en découlent ont pour vocation non pas de représenter fidèlement la réalité d'une situation, ce qui s'apparente au sens de Lacroux (1999) à une recherche du «vrai », mais de permettre l'expression de son interprétation par les acteurs de l'entreprise pour faciliter la compréhension de la complexité à des fins d'action. La modélisation permet donc la construction d'artefacts évolutifs favorisant la mise en œuvre de projets pour l'action humaine (Lorino, 1999). Ce qui revient à dire que la manière de poser le problème conditionne le cheminement de la solution. La modélisation a pour objectif, non seulement de comprendre la situation, mais aussi de faciliter la communication entre les membres de l'entreprise et les intervenants-modélisateurs dans une perspective de création de valeur. Les résultats qui en découlent doivent être physiquement cohérents, intellectuellement accessibles et socialement acceptables.

En d'autres termes, notre recherche s'inscrit dans un cadre épistémologique constructiviste, dans la mesure où l'on ne cherche pas à connaître la réalité, «mais

8. L'idée de construction est à contraster avec celle d'adaptation.

Revue internationale P.M.E., vol. 15, nº 2, 2002 
à mieux comprendre comment les représentations se construisent et de quelle manière elles peuvent servir à atteindre des finalités pragmatiques » (Yatchinovsky, 1999).

L'idée de modélisation oblige à redéfinir le rôle de l'intervenant. Ainsi, ce dernier n'est plus là seulement pour apporter une solution à un problème donné, il a aussi pour mission de faciliter la représentation des problèmes, c'est-à-dire la modélisation des situations complexes. Dans ces conditions, l'objectif est de développer des savoirs sur les processus à partir de raisonnements heuristiques ${ }^{9}$ visant à fournir une aide à la construction de problèmes au sein des PME.

De façon plus synthétique, l'articulation du rôle de l'intervenant peut se résumer par la métaphore des planètes Alpha et Bêta de Caillé (1991):

- sur Alpha, chaque organisation est dotée d'un « engin » qui fournit automatiquement les réponses adéquates à tous les problèmes qui peuvent surgir (raisonnement algorithmique). Lorsque cet engin est en panne, l'organisation fait appel à un «réparateur» qui assurera les réglages nécessaires et remplacera les éléments défectueux;

- sur Bêta, chaque organisation construit ses outils avec les moyens dont elle dispose (raisonnement heuristique). Ces organisations peuvent, en cas de difficulté, faire appel à un «facilitateur». Le «facilitateur» ne vient pas apporter la solution; il se sert avant tout de sa position pour aider le système à se donner une représentation réflexive de lui-même, à ne pas s'enfermer dans son point de vue, à percevoir ce qui est peut-être autrement, à redevenir acteur et créateur de son devenir.

Il convient donc de rétablir ce rôle de facilitateur, trop souvent oublié dans les interventions en PME, aux côtés du rôle traditionnel de réparateur. L'intervenant devient alors modélisateur. Soulignons également que la gestion des problèmes complexes nécessite avant tout un rôle de facilitateur et que le rôle de réparateur y est subordonné.

Dans ces conditions, il convient de s'interroger pour savoir comment traduire en termes de méthode d'intervention le cadre épistémologique retenu, c'est-à-dire trouver « des clés capables (ou non) d'ouvrir certaines serrures susceptibles (ou non) de convenir pour organiser et faire évoluer une situation» (Roy, 1992) dans le but «d'amener les individus à prendre conscience de leurs comportements habituels afin de les modifier » (Argyris, 1995).

9. Il s'agit de raisonnements qui, vraisemblablement, peuvent conduire à la découverte de solutions satisfaisantes au problème.

Revue internationale P.M.E., vol. 15, nº 2, 2002 


\section{Pratiques et résultats de la modélisation des problèmes complexes}

À la lumière des différents constats faits jusqu'ici, l'objectif de cette partie est donc de représenter les interactions des variables du système étudié. Pour cela, nous avons eu recours à une approche matricielle, approche qui offre la possibilité « de décrire un système à l'aide d'une matrice mettant en relation les éléments constitutifs du système » (Hatam, 1993). Elle s'inspire de travaux variés comme la théorie des graphes (Avenier, 1990), la théorie du système général (Le Moigne, 1977) ou encore les travaux de prospective (Lefebvre, 1982; Godet, 1984).

\subsection{Proposition d'une instrumentation pour gérer les problèmes complexes}

La représentation des connexions de plusieurs variables, agencées en réseau, se révèle en pratique extrêmement aisée (Le Moigne, 1977) et favorise la mise en lumière de la structure des relations entre les variables qui caractérisent la situation étudiée (Godet, 1984). Dans notre cas, l'utilisation de matrices vise à étudier, en termes d'influence et de dépendance, les relations entre les variables et à faire émerger celles qui sont essentielles à la compréhension des situations problématiques. Dans cette perspective, la démarche que nous avons élaborée comporte trois étapes.

1) La définition d'une cible et de variables. Dans un premier temps, il est nécessaire de définir une cible d'observation. Elle correspond à l'orientation stratégique établie ou à établir par le dirigeant de la PME. Cette cible donne un sens à la lecture des représentations graphiques ultérieures. La définition de cette cible permet de dégager les variables ainsi qu'une cohérence entre elles; ces variables correspondent à des facteurs clés de succès, c'est-à-dire à des variables essentielles pour la réalisation des orientations stratégiques.

2) Le remplissage de la matrice. L'objectif est de remplir une matrice carrée à partir des variables retenues précédemment. Il s'agit ici de trouver les relations directes entre les variables. Pour cela, il convient de répondre à la question générique suivante : «Actuellement, dans l'entreprise A, existe-t-il une influence directe entre le facteur $\mathrm{X}$ et le facteur $\mathrm{Y}$ ? » Les réponses à ces multiples questions sont données par les différents intervenants ayant participé à la collecte d'informations dans l'entreprise. En ce sens, ce travail est un exercice de communication et de réflexion collective. Le remplissage de la matrice est qualitatif : 0 , s'il n'existe pas de relation entre $\mathrm{X}$ et $\mathrm{Y}$; 1 , pour une influence faible ; 2 , pour une influence moyenne ; 3 , pour une influence forte. À partir de cette matrice, il est possible de faire la somme des colonnes (dépendance) et des lignes (influence).

Revue internationale P.M.E., vol. 15, n 2, 2002 
3) La mise en évidence des variables clés. La sommation en lignes et en colonnes donne les coordonnées des différents facteurs. Ces points sont placés dans un plan influence-dépendance, appelé schéma topographique. La lecture du graphique se fait à partir de deux lignes de démarcation permettant de dégager quatre secteurs ou quatre quadrants (l'influence, dans le quadrant nord-ouest, les enjeux dans le quadrant nord-est, la dépendance dans le quadrant sud-ouest et l'autonomie dans le quadrant sud-est). Cette démarcation est calculée en fonction du poids des facteurs. Le schéma topographique est donc un schéma unique correspondant à une entreprise particulière.

FIGURE 2

\section{Schéma topographique : représentation et compréhension}

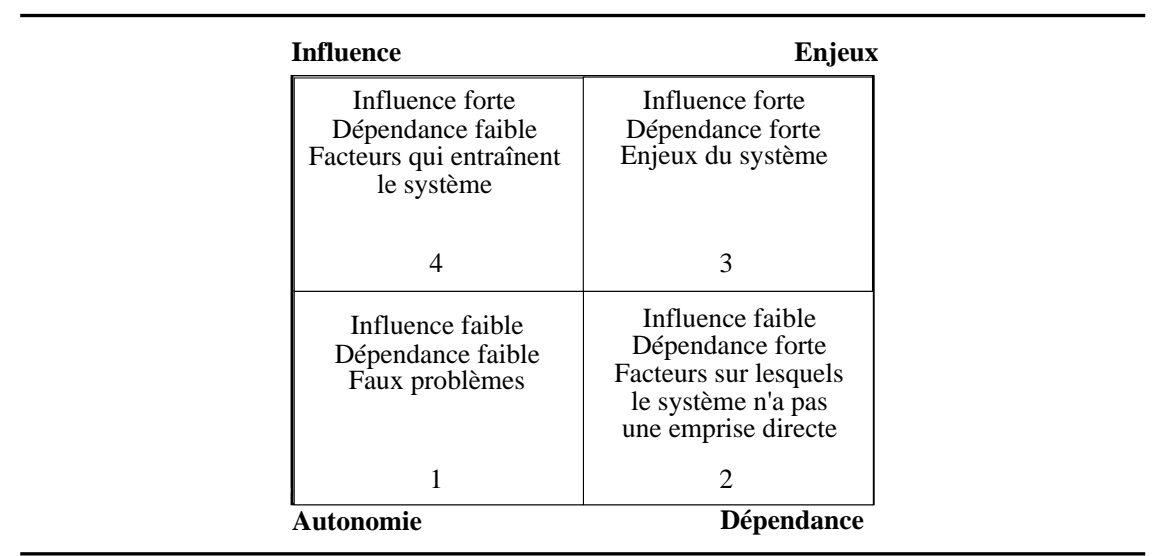

\section{Lecture d'un schéma topographique (d'après Godet, 1984)}

On peut distinguer quatre secteurs ou quadrants :

- les variables du secteur 1 correspondent à des variables ayant peu de relations avec le système. Elles ne sont donc pas dynamiques ou le sont peu ;

- les variables du secteur 2 sont avant tout dépendantes, donc susceptibles d'évoluer. Le positionnement de ces variables s'explique par l'influence des variables des secteurs 3 et 4 ;

- les variables du secteur 3 sont très influentes et très dépendantes, elles doivent donc faire l'objet d'une attention particulière. Leur évolution a des incidences sur le reste du système et sur elles-mêmes;

- les variables du secteur 4 conditionnement le reste du système par l'importance de leur influence. 


\subsection{Le cadre d'intervention: les études de caractérisation de la Chaire Bombardier}

Le travail mené sur la modélisation des problèmes complexes en PME s'inscrit dans les études de caractérisation menées par un centre de recherche québécois dont l'un des objectifs est d'aider des PME sous-traitantes à passer dans la classe mondiale pour mieux répondre aux besoins d'un grand donneur d'ordres (Jacob, Julien et Raymond, 1996). La vocation de ce centre est, de plus, de créer un réseau synergique d'entreprises fondé sur le développement, l'implantation et le transfert, en contexte de partenariat, de nouvelles technologies opérationnelles et organisationnelles. Dans cette perspective, ces études de caractérisation ont pour but d'aider les PME dans la mise sur pied de démarches visant à intégrer systématiquement la recherche de valeur dans leurs opérations. Par exemple, la mise en place d'un système de flux tendus (juste à temps) en contexte de production à valeur ajoutée (JAT/PVA) a été relevée comme un des problèmes complexes actuels des PME. En effet, une étude récente menée auprès d'un échantillon de 406 PME (Carrière, 1995) montre que $45,2 \%$ des entreprises sous-traitantes appliquent à différent niveau le JAT. On mentionne, cependant, que cette technologie d'intégration est surtout utilisée pour la gestion des stocks et beaucoup moins dans une perspective globale d'amélioration des procédés organisationnels et opérationnels. D'un autre point de vue, des travaux récents portant sur les défis de la compétitivité au Québec (Martel et Oral, 1995) indiquent clairement qu'un des plus grands défis des entreprises du Québec réside dans leur capacité à mettre en place une gestion et une production de classe mondiale. Les auteurs s'appuient notamment sur l'exemple de l'intégration du JAT à production à valeur ajoutée.

Le JAT à production à valeur ajoutée joue donc le rôle de cible ou d'orientation stratégique permettant de définir les facteurs qui lui sont associés afin de les classer et de les organiser en grilles d'analyse de l'état des entreprises par rapport au JAT. Sur la base d'une analyse de la documentation la plus complète possible (recherches empiriques, études de cas ou analyses conceptuelles), 54 facteurs de succès du JAT ont été identifiés et retenus. Ces facteurs peuvent être regroupés en 10 catégories (annexe I) appartenant à deux classes : les facteurs organisationnels et les facteurs opérationnels. Cette approche est beaucoup plus complète que les approches classiques à une classe, la plupart du temps opérationnelle et basée sur la gestion des stocks.

Actuellement, une vingtaine d'études de caractérisation ont été menées par les chercheurs de la Chaire Bombardier auprès des firmes partenaires sous-traitantes de la firme Bombardier Produits récréatifs. Dans un souci d'amélioration continue de ces études, un projet de modélisation des problèmes complexes à partir du JAT a été élaboré afin de compléter les analyses actuelles portant sur les facteurs clés de succès (figure 3 ). L'objectif est quadruple, il s'agit de :

Revue internationale P.M.E., vol. 15, $\mathrm{n}^{\circ}$ 2, 2002 
- construire une représentation globale de la PME par rapport au JAT ;

- faire émerger les facteurs clés confirmant ou non l'analyse des facteurs ;

- faciliter l'agencement d'un diagramme de préséance regroupant les différentes recommandations selon un ordre logique temporel de façon à tenir compte de changements préalables nécessaires à des changements plus importants par la suite;

- contribuer à la connaissance du processus stratégique en PME pouvant servir tant aux entreprises qu'aux chercheurs.

Cette démarche vise donc à fournir des clés de compréhension nouvelles des situations complexes pour les appréhender et entreprendre des actions efficaces.

FIGURE 3

Le processus d'intervention en PME



\subsection{Présentation des principaux résultats}

Cette démarche de modélisation a été utilisée dans une PME de production industrielle regroupant 74 personnes. L'intervention s'est déroulée en cinq phases (formation du personnel clé de l'entreprise, collecte de données à l'aide des documents existants et auprès d'un grand nombre d'employés et de cadres de l'entreprise à l'aide de questionnaires et de grilles d'entrevues, analyse de ces données, élabora- 
tion de recommandations et préséance de celles-ci, présentation du rapport) pour une durée totale de 20 semaines $^{10}$. La démarche de modélisation s'est basée sur l'information déjà recueillie pour l'analyse des 54 facteurs. De plus, l'utilisation simultanée de ces deux approches permet de voir les correspondances d'analyse entre les deux. Ainsi, dans ce cas, 50 entretiens semi-directifs ont été effectués, la moitié sur l'aspect organisationnel et l'autre sur l'aspect opérationnel.

Une fois la matrice remplie collectivement avec l'équipe d'intervention à l'aide des informations issues de l'analyse des facteurs, nous avons pu dégager les grands traits de l'organisation de la PME à partir du schéma topographique suivant ${ }^{11}$ :

FIGURE 4

Exemple de lecture : le schéma topographique de la PME étudiée

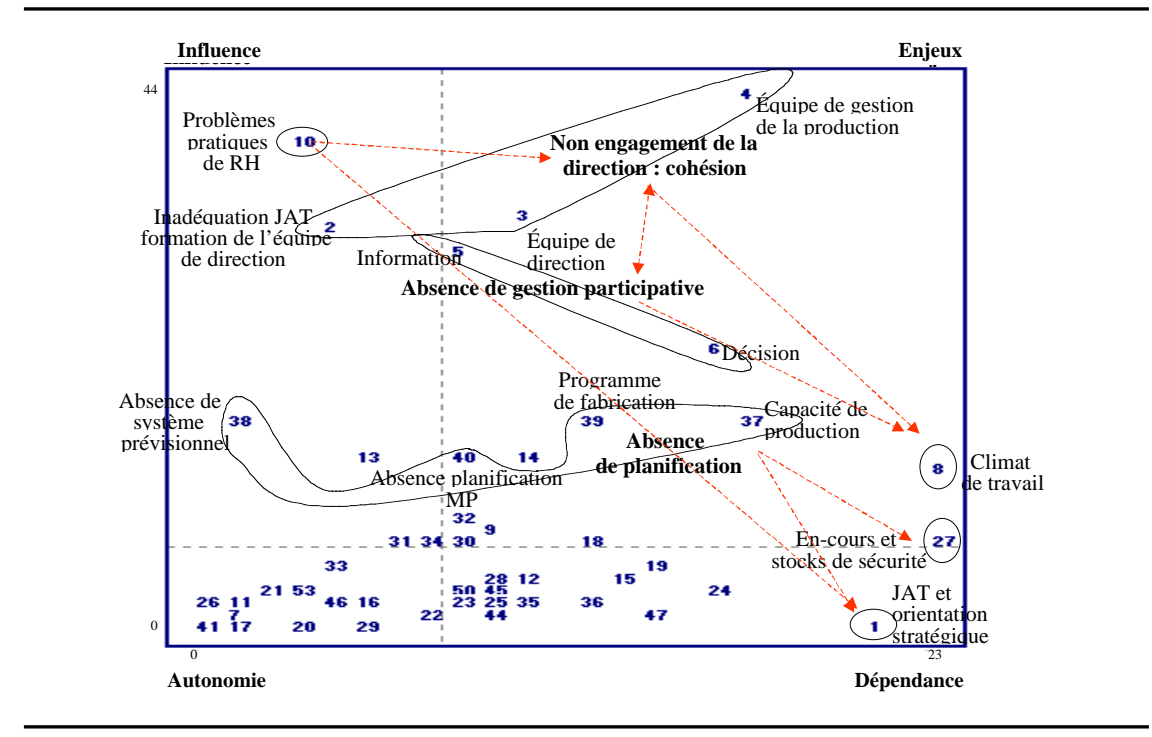

La construction du schéma topographique fait émerger quatre secteurs.

Secteur 1 (quadrant sud-ouest) : autonomie. Il s'agit principalement de facteurs opérationnels ; ces facteurs influencent peu le système et sont peu influencés par lui. À eux seuls, ils représentent $35 \%$ des facteurs. Ainsi, les actions menées

10. Cela correspond à la durée moyenne d'une étude de caractérisation. La construction d'un schéma topographique a été réalisée lors d'une réunion de quatre heures.

11. Pour ne pas surcharger le graphique, certains facteurs n'apparaissent pas, car ils ont les mêmes coordonnées que d'autres points. Pour la position de tous les facteurs, voir l'annexe I. 
sur ces facteurs n'auront qu'une incidence locale sur l'entreprise. Le développement d'une logique de JAT à partir de ces facteurs doit être considéré comme un faux problème.

Secteur 2 (quadrant sud-est) : dépendance. Ces facteurs principalement opérationnels sont fortement influencés par les facteurs des secteurs 3 et 4 . Ils sont le résultat de l'évolution des facteurs plus influents et représentent $28 \%$ des facteurs étudiés.

Secteur 3 (quadrant nord-est) : enjeux. Ils correspondent à $26 \%$ de la totalité des facteurs. Ils sont autant influencés qu'influençables. Ils sont en constante évolution, ils sont donc par nature instables. En effet, les actions menées sur ces facteurs par l'entreprise ont « des répercussions sur les autres et sur elles-mêmes » (Godet, 1984). En ce sens, ces facteurs représentent des occasions favorables ou des menaces potentielles pour l'entreprise.

Secteur 4 (quadrant nord-ouest) : influence. Ces facteurs, qui ne représentent que $11 \%$ de la totalité des facteurs, conditionnent le reste du système; ils permettent d'expliquer la position des autres facteurs. Toute action dans une perspective de mise en place d'un système de JAT en contexte de production à valeur ajoutée nécessite de tenir compte de ces facteurs.

À partir de cette lecture par secteur, nous avons pu relever les facteurs clés de l'organisation, tant du point de vue de l'influence que de la dépendance. Dans la mesure du possible, nous les avons regroupés par rapport aux catégories initiales. La suite de l'article propose quelques exemples de lecture.

Les facteurs les plus influents du système s'organisent essentiellement autour de six facteurs organisationnels (facteurs 2, 3, 4, 5, 6 et 10; voir l'annexe I). À eux seuls, ils expliquent près de $50 \%$ des influences de la PME étudiée. Signalons que ces facteurs se caractérisent par leur absence relative dans l'entreprise. Ainsi, les difficultés que connaît cette entreprise s'apparentent à un trop faible engagement de la direction (facteurs 2,3 et 4 ) et à une absence de gestion participative (facteurs 5 et 6 , correspondant à un faible partage de l'information et des décisions et à une absence de rémunération incitative). De plus, les problèmes actuels de l'entreprise sont liés au manque de formalisation de la fonction ressource humaine et au climat de travail malsain (facteurs 8 et 10). Cela se caractérise sur le terrain par un changement important du personnel, notamment sur le plan organisationnel (taux de roulement de 40,7\% en 1999 et de $28,1 \%$ pour le premier semestre 2000). Cela entraîne non seulement un coût élevé de formation et de recrutement, mais surtout une perte importante de savoirfaire. Ces facteurs ont une influence autant sur les plans organisationnel qu'opérationnel.

En ce qui concerne les facteurs les plus dépendants, ils sont au nombre de 13 (soit ceux qui représentent les plus fortes dépendances); ils correspondent autant à des facteurs organisationnels qu'opérationnels. Les facteurs les plus pertinents

Revue internationale P.M.E., vol. 15, nº 2, 2002 
sont les facteurs 1,27 et 8. Les deux premiers facteurs correspondent, dans un mode de gestion basé sur le JAT, à des facteurs qui devraient être plus influents ; or, ici, ils apparaissent comme des facteurs résultats sur lesquels le système n'a pas d'emprise, car influencés par d'autres facteurs. Plus précisément, le facteur 1 correspond à l'adéquation entre le projet JAT et les orientations stratégiques; ces dernières existent et sont partagées au sein de la PME, mais ne sont visiblement pas mises en application. Cela se traduit sur le terrain par une stratégie à court terme influencée par une absence de planification (facteurs 37, 38, 39 et 40) et le manque de formalisation de la direction des ressources humaines (facteur 10). Le facteur 27 , minimisation des en-cours et des stocks de sécurité, est, quant à lui, fortement influencé par les difficultés liées à l'absence de planification (facteurs 37, 38, 39 et 40). Enfin, le facteur 8, climat de relations de travail, est fortement influencé par les difficultés éprouvées par l'entreprise, c'est-à-dire le manque d'engagement et de cohésion de la direction (facteurs 2,3 et 4) et l'absence de gestion participative (facteurs 5 et 6). Cela se caractérise notamment par un climat d'insécurité (p. ex., les rumeurs), la peur de prendre des décisions, l'individualisme ou, encore, l'absence de reconnaissance et l'ignorance des suggestions d'amélioration.

En résumé, la lecture du graphique nous révèle que les problèmes de l'entreprise, par rapport à l'objectif de JAT en contexte de production à valeur ajoutée, seraient avant tout de nature organisationnelle. Cela se traduit dans les faits par un non-engagement de la direction dans le projet, l'absence de gestion participative, l'absence de formation et des relations de travail difficiles; ce qui montre bien que des actions de base portant par exemple sur une meilleure gestion des stocks ne sont pas suffisantes. De plus, ce premier niveau de lecture est complété par une étude plus approfondie des relations entre les différents facteurs afin d'élaborer des recommandations réunies dans un diagramme de préséance priorisant les actions stratégiques à mener autour notamment d'un engagement de la direction.

\section{Discussions et perspectives}

\subsection{Discussions}

En reprenant la définition de la modélisation ${ }^{12}$ donnée par Le Moigne (1990), il apparaît que l'outil utilisé permet de concevoir des représentations rendant intelligibles les problèmes complexes et favorisant la communication entre intervenants et praticiens. Dans cette perspective, ce genre d'outils a une triple portée :

12. Le Moigne (1990) caractérise la modélisation comme «une action d'élaboration et de construction intentionnelle, par composition de symboles, de modèles susceptibles de rendre intelligible un phénomène perçu comme complexe et d'amplifier le raisonnement de l'acteur projetant une intervention délibérée au sein du phénomène ; raisonnement visant notamment à anticiper les conséquences de ces projets d'actions possibles ».

Revue internationale P.M.E., vol. 15, nº 2, 2002 
- elle favorise, dans un premier temps, un travail de consensus chez les intervenants en vue de construire une représentation permettant de faire réfléchir à des aspects contre-intuitifs. En ce sens, il ne faut pas oublier que le schéma topographique n'est que la représentation des intervenantsmodélisateurs de la représentation de la réalité qu'ont les acteurs (Verstraete, 1997);

- chez les praticiens, les outils basés sur le visuel permettent d'avoir une image globale de la situation problématique et, par conséquent, de modifier leur propre représentation, faisant même émerger des liens jusque-là ignorés et pour certains inenvisageables ultérieurement. Comme le souligne Godet (1984), il convient de rappeler ici que l'objectif de la modélisation n'est pas de prendre la place du décideur en prétendant décrire avec précision le fonctionnement de l'entreprise, mais bien d'aider les PME à s'inscrire dans un processus d'amélioration continue ;

- enfin, les outils utilisés se caractérisent par leur aspect visuel ${ }^{13}$. Ils agissent comme un outil de communication entre les intervenants et les praticiens. Les schémas utilisés pour modéliser les situations complexes ne sont pas seulement descriptifs, ils visent également à mieux se représenter et à simuler ce qui peut se passer dans l'avenir. Il s'agit alors de construits intentionnels ou d'artefacts évolutifs servant à appréhender la situation qu'ils servent à représenter. En ce sens, on parlera d'outils d'aide à la représentation. Le mode de représentation réticulaire que nous avons adopté a donc une portée largement stratégique (Nicot, 1997); il fait figure de «langage universel du fait de la rapidité de la perception visuelle ainsi que de la simultanéité apparente de la reconnaissance de son contenu et de son interprétation » (Joly, 1993). À ce titre, on peut dire de lui qu'il a un effet espéranto, car il permet d'utiliser les divers langages du personnel pour en arriver à une meilleure compréhension de la situation.

\subsection{Perspectives: vers le développement d'une recherche ingénierique en PME}

À partir de cette proposition, il paraît intéressant d'entreprendre des démarches de modélisation des problèmes complexes et des outils d'aide à la construction des problèmes. En effet, comme nous l'avons souligné précédemment, les PME ne

13. L'utilisation d'outils visuels n'est certes pas une idée innovante; mais force est de constater le peu d'intérêt que cela suscite dans la recherche en science de l'organisation en général et en gestion, en particulier (Servant, 1990). Pourtant, les plaidoyers pour l'utilisation de modes de représentations graphiques ne manquent pas. Ils peuvent se résumer par la question, apparemment banale, posée par Simon (1991) : «Comment se fait-il qu'un modeste schéma nous en dise souvent plus qu'un long discours?»

Revue internationale P.M.E., vol. 15, nº 2, 2002 
disposent pas d'outils permettant de rendre intelligible la complexité ou très peu. Il s'agit donc de concevoir des démarches d'application scientifique et d'étude globale d'un problème qualifié de complexe sous tous ces aspects (opérationnels et organisationnels); on parlera alors de recherche ingénierique. Comme le souligne Martinet (1996), ce type de recherche

[...] montre l'un des genres possibles que peuvent revêtir les sciences de gestion quand on les envisage plutôt sous l'angle de l'ingénierie, de la conception ou de ce qu'avec le Prix Nobel Herbert A. Simon l'on peut appeler les sciences de l'artificiel. Car il s'agit bien de connaître suffisamment la réalité pour mieux l'inventer. [...] Ce genre de recherche débouche sur une pensée-méthode susceptible d'aider l'acteur à bien conduire sa raison, à se saisir des situations complexes qu'il doit affronter et à instruire des choix en meilleure connaissance de cause comme de finalité et de conséquences.

La recherche ingénierique associe à la fois les connaissances théoriques concernant l'approche des situations complexes et « une appropriation des résultats de la recherche par des praticiens, ce qui est une dimension fondamentale de l'apprentissage organisationnel » (Chanal, Lesca et Martinet, 1997).

Différents travaux contribuent déjà indirectement au développement d'une recherche ingénierique en PME. C'est le cas, notamment, des travaux basés sur les cartes cognitives, comme on l'a dit. Parallèlement à cela, les sciences pour l'ingénieur ainsi que la gestion des opérations utilisent des méthodologies, telles que l'analyse de la valeur ou encore les outils de la qualité, diagramme de Pareto ou diagramme Ishikawa, pouvant être, dans une certaine mesure, des outils transférables pour l'approche des problèmes complexes (Michel, 1997; Schmitt et Grandhaye, 1999-2000). Ainsi, la recherche ingénierique oriente la gestion vers une approche plus expérimentale.

Au final, comme le soulignent Chanal, Lesca et Martinet (1997) «la valeur scientifique n'est pas à chercher du côté de la validité statistique qui nécessite un grand nombre de données. [...] La recherche ingénierique, qui ne peut évidemment travailler que sur un nombre limité de cas, privilégie au contraire l'exploration en profondeur et la durée ».

\section{Conclusion}

La complexité ne peut plus être qu'un simple postulat de recherche. Elle nécessite, comme le soulignent Morin et Le Moigne (1999), non seulement de «relier, de contextualiser, de globaliser des situations qui ne correspondent plus à l'entendement habituel», mais aussi de concevoir des outils favorisant l'action. La complexité des situations à gérer est donc une réalité incontournable en PME. Dans cette perspective, les apports de notre recherche s'articulent autour de deux points :

Revue internationale P.M.E., vol. 15, $\mathrm{n}^{\circ}$ 2, 2002 
- le développement de nouvelles connaissances en portant un regard différent sur les actions menées en PME. Les problèmes de gestion de la complexité peuvent engendrer des situations paradoxales où la solution est le problème. Ces difficultés se comprennent à la lumière des spécificités des PME (la centralisation de la gestion, une stratégie intuitive ou peu formalisée, l'absence ou la quasi-absence d'outils pour aborder la complexité des situations). L'intérêt est donc de constater sa présence au niveau de la PME, impliquant le recours à des méthodes et à des outils appropriés. Ce paradoxe n'est pas nouveau et s'inscrit dans le développement «d'une théorie de la PME de l'instabilité » (Julien, 1989) où la PME est envisagée en constante évolution;

- la proposition d'une démarche pour rendre intelligible la complexité à partir d'un cadre épistémologique constructiviste. L'originalité de notre proposition repose sur le fait que nous soumettons un cadre d'intervention axé sur le visuel (Adam, 1999). Ce support permet de recadrer la situation, c'est-à-dire de changer le regard que posent les acteurs sur une situation donnée. Ce qui implique que «l'acteur doit faire preuve de cette forme d'intelligence qui consiste moins en la résolution d'un problème qu'en la mise en forme d'un monde partageable» (Martinet, 1993). En d'autres termes, la complexité peut se comprendre par le visuel.

L'expérience de modélisation présentée dans cet article, ainsi que le témoignage des personnes ayant participé au projet, chercheurs, intervenants et praticiens, confirme que la méthodologie utilisée va dans le sens d'une meilleure connaissance des problèmes complexes qui surviennent dans les PME. Il semble donc vital de continuer à mettre en place des outils permettant la construction des problèmes et une meilleure représentation de ceux-ci, car, comme le rappelle Delorme (1999), bien que la frontière entre complexe et non complexe ne soit pas absolue, «il est plus opératoire de faire comme si la situation était potentiellement complexe quitte ensuite à reconnaître sa non-complexité».

En conclusion, le développement d'une recherche ingénierique en PME doit favoriser l'articulation entre les connaissances à propos de l'action et les connaissances pour l'action afin de comprendre la réalité d'aujourd'hui et construire celle de demain.

Revue internationale P.M.E., vol. 15, nº 2, 2002 


\section{AnNeXe I \\ Facteurs utilisés pour mettre en place un système de flux tendus (juste à temps) en contexte de production à valeur ajoutée par la Chaire Bombardier}

\begin{tabular}{|c|c|}
\hline \multicolumn{2}{|c|}{ Facteurs organisationnels } \\
\hline \multicolumn{2}{|c|}{ Management : Engagement de la direction } \\
\hline F1 & Adéquation entre le projet JAT et l'orientation stratégique \\
\hline $\mathrm{F} 2$ & $\begin{array}{l}\text { Adéquation entre le projet JAT et la formation professionnelle } \\
\text { de l'équipe de direction }\end{array}$ \\
\hline F3 & Cohésion de l'équipe de direction \\
\hline $\mathrm{F} 4$ & Cohésion de l'équipe de gestion des opérations \\
\hline \multicolumn{2}{|c|}{ Management: Gestion participative } \\
\hline F5 & Au niveau de l'information \\
\hline F6 & Au niveau de la décision \\
\hline F7 & Au niveau de l'avoir \\
\hline \multicolumn{2}{|c|}{ Relations de travail } \\
\hline F8 & Climat de relation de travail \\
\hline F9 & Mécanismes de gestion des différends \\
\hline F10 & Formalisation de la direction des ressources humaines \\
\hline \multicolumn{2}{|c|}{ Formations } \\
\hline F11 & Conception stratégique de la formation par la direction générale \\
\hline F12 & Formation qualifiante \\
\hline F13 & Plan de formation \\
\hline F14 & Budget consacré \\
\hline F15 & Formation continue \\
\hline \multicolumn{2}{|c|}{ Rémunération } \\
\hline F16 & Équité interne \\
\hline F17 & Équité externe \\
\hline \multicolumn{2}{|c|}{ Main-d'œuvre } \\
\hline F18 & Capacité d'adaptation au changement \\
\hline F19 & Flexibilité de la main-d'œuvre \\
\hline \multicolumn{2}{|c|}{ Facteurs opérationnels } \\
\hline \multicolumn{2}{|c|}{ Gestion de l'approvisionnement } \\
\hline F20 & Coopération et climat de confiance avec les fournisseurs \\
\hline $\mathrm{F} 21$ & Évaluations des fournisseurs \\
\hline $\mathrm{F} 22$ & Contrats à long terme basés sur l'exclusivité ou le partenariat \\
\hline $\mathrm{F} 23$ & Communication du programme de production aux fournisseurs \\
\hline $\mathrm{F} 24$ & Élaboration d'une stratégie d'achat \\
\hline $\mathrm{F} 25$ & Efficacité et efficience du service des achats \\
\hline $\mathrm{F} 26$ & Efficacité du réseau de communication \\
\hline
\end{tabular}


ANNEXe I (suite)

Facteurs utilisés pour mettre en place un système de flux tendus (juste à temps) en contexte de production à valeur ajoutée par la Chaire Bombardier

\begin{tabular}{cl}
\hline Gestion des stocks \\
F27 & Minimisation des en-cours et des stocks de sécurité \\
F28 & Inventaire en continu et informatisé \\
F29 & Contrôle des expéditions et du transport \\
\hline Production \\
F30 & Minimisation de la taille des lots de production \\
F31 & Minimisation des lots de transfert \\
F32 & Réduction des temps d'opération et de mise en course \\
F33 & Méthodes et mesure du travail \\
F34 & Standardisation de la production \\
F35 & Développement et amélioration des produits et des procédés \\
\hline F36 & Efficacité et efficience des procédés \\
F37 & Maîtrise et gestion de la capacité de production \\
F38 & Fiabilité du système prévisionnel \\
F39 & Programme de fabrication \\
F40 & Planification des besoins de matières \\
F41 & Programme d'entretien préventif et fiabilité des équipements \\
F42 & Système informatique flexible, adaptable et adéquat \\
\hline Aménagement de l'usine \\
F43 & Flexibilité de la production et aménagement cellulaire \\
F44 & Autonomie organisationnelle des ateliers ou des cellules \\
F45 & Aménagement de l'usine \\
F46 & Aménagement des postes de travail \\
F47 Élimination de la manutention \\
F48 & Santé, sécurité et hygiène industrielle \\
\hline Qualité & \\
F49 & Groupe d'amélioration de la qualité \\
F50 Autocontrôle aux postes de travail \\
F51 Contrôle par échantillonnage des ateliers et contrôle statistique des procédés \\
F52 Certification de la qualité \\
F53 Spécification du niveau de non-qualité et de surqualité \\
F54 Structure organisationnelle de l'assurance qualité \\
\hline
\end{tabular}

Revue internationale P.M.E., vol. 15, $\mathrm{n}^{\circ}$ 2, 2002 
ANNEXE II

Coordonnées des facteurs dans le plan influence-dépendance

\begin{tabular}{crrccc}
\hline Facteurs & Dépendance & Influence & Facteurs & Dépendance & Influence \\
\hline FI & 21 & 0 & F28 & 9 & 4 \\
F2 & 4 & 33 & F29 & 5 & 0 \\
F3 & 10 & 34 & F30 & 8 & 7 \\
F4 & 17 & 44 & F31 & 6 & 7 \\
F5 & 8 & 31 & F32 & 8 & 9 \\
F6 & 16 & 23 & F33 & 4 & 5 \\
F7 & 1 & 1 & F34 & 7 & 7 \\
F8 & 23 & 13 & F35 & 10 & 2 \\
F9 & 9 & 8 & F36 & 12 & 2 \\
FIO & 3 & 40 & F37 & 17 & 17 \\
F11 & 1 & 2 & F38 & 1 & 17 \\
F12 & 10 & 4 & F39 & 12 & 17 \\
F13 & 5 & 14 & F40 & 8 & 14 \\
F14 & 10 & 14 & F41 & 0 & 0 \\
F15 & 13 & 4 & F42 & 0 & 0 \\
F16 & 5 & 2 & F43 & 9 & 2 \\
F17 & 1 & 0 & F44 & 9 & 1 \\
F18 & 12 & 7 & F45 & 9 & 3 \\
F19 & 14 & 5 & F46 & 4 & 2 \\
F20 & 3 & 0 & F47 & 14 & 1 \\
F21 & 2 & 3 & F48 & 0 & 0 \\
F22 & 7 & 1 & F49 & 9 & 1 \\
F23 & 8 & 2 & F50 & 8 & 3 \\
F24 & 16 & 3 & F51 & 0 & 0 \\
F25 & 9 & 2 & F52 & 0 & 0 \\
F26 & 0 & 2 & F53 & 3 & 3 \\
F27 & 23 & 7 & F54 & 0 & 0 \\
\hline
\end{tabular}

\section{Bibliographie}

ADAM, M. (1999), Les schémas, un langage transdisciplinaire. Les comprendre, les réussir, Paris, L'Harmattan.

ARGYRIS, C. (1995), Savoir pour agir, surmonter les obstacles à l'apprentissage organisationnel, Paris, InterÉditions.

AVENIER, M.-J. (1988), Le pilotage stratégique de l'entreprise, Paris, Presses du CNRS.

AVENIER, M.-J. (1990), «Apports d'un système interactif de représentations graphiques hiérarchisées, pour l'intelligence de phénomènes complexes », $\mathrm{CXV}^{\mathrm{e}}$ Congrès national des sociétés savantes, Avignon, L'image et la science, p. 285-302.

BAREL, Y. (1979), Le paradoxe et le système, Grenoble, Presses universitaires de Grenoble. 
BAYAD, M. et A. PARADAS (1998), «Difficultés de recrutement en PME: recherche sur les déterminants organisationnels », dans O. Torrès (dir.), PME, de nouvelles approches, Paris, Economica, p. 145-160.

BAYAD, M. et D. NEBENHAUs (1998), «Contribution à un modèle pyramidal de la gestion des ressources humaines en PME », Revue internationale PME, vol. 11, $\mathrm{n}^{\text {os }} 2-3$, p. $161-178$.

BERGANDI, D. (1998), «Les antinomies épistémologiques entre les réductionnistes et les émergentistes », Revue internationale de systémique, vol. 12, nº 3, p. 225-252.

BLACKSTONE, J.H. et J.F. COX (1985), « Are small manufacturers ready for MRP ? », Comptes rendus de la XXVII e conférence internationale annuelle de l'APICS, p. 3-6.

CAILlé, P. (1991), Un et un font trois, Paris, ESF.

CARRIÈRE, J.B. (1995), Profil technologique de la PME manufacturière au Québec, 1995, Rapport de recherche, Québec, CEFRIO.

Chanal, V., H. Lesca et A.C. Martinet (1997), « Vers une ingénierie de la recherche en sciences de gestion », Revue française de gestion, $\mathrm{n}^{\circ} 116$, novembre-décembre, p. 41-51.

Cossette, P. (dir.) (1994), Cartes cognitives et organisations, Québec, Les Presses de l’Université Laval ; Paris, Eska.

DAMANPOUR, F. (1996), «Organizational complexity and innovation: developing and testing multiple contingency models », Management Science, vol. 42, $\mathrm{n}^{\circ}$ 5, p. 693-716.

DELOBEL, B. (1998), «La PME, un mode (très adapté) de réponse aux événements. Contribution à une théorie de l'instabilité/stabilité de la PME», Actes du IVe Colloque international francophone de la PME, Nancy-Metz; actes sur CD-ROM.

DELORME, R. (1999), «De l'emprise à l'en-prise. Agir en situations complexes », dans Entre systémique et complexité, chemin faisant, Paris, Presses universitaires de France, p. 27-46.

FABI, B. (1997), «La gestion des ressources humaines en PME», dans P.-A. Julien (dir.), Les PME : Bilan et perspectives, Paris, Economica, p. 255-303.

FILION, L.J. (1991), Visions et relations, Montréal, Éditions de l'Entrepreneur.

GÉlinas, R., A. HALley, R. JACOB et J. Drolet (1996), «Les caractéristiques et les spécificités de la PME : favorables ou défavorables au juste-à-temps », Revue internationale PME, vol. 9, $\mathrm{n}^{\circ}$ 2, p. 81-101.

GÉNELOT, D. (1992), Manager dans la complexité. Réflexions à l'usage des dirigeants, Paris, Éditions Insep.

Gervais, M. (1991), Contrôle de gestion et stratégie de l'entreprise, 4e édition, Paris, Economica.

Revue internationale P.M.E., vol. 15, $\mathrm{n}^{\circ}$ 2, 2002 
GIRIN, J. (1990), «L'analyse empirique des situations de gestion : éléments de théorie et de méthodes », dans A.-C. Martinet (dir.), Épistémologies et sciences de gestion, Paris, Economica, p. 141-181.

Girod-SÉviLle, M. et V. PERret (1999), «Fondements épistémologiques de la recherche », dans R.A. Thietart (dir.), Méthode de recherche en management, Paris, Dunod, p. 13-33.

Godet, M. (1984), Prospective et planification stratégique, Paris, Economica.

GodET, M. (1994), «La tête dans la vision globale, les pieds dans la glaise locale», Futuribles, mai, p. 45-57.

HATAM, F. (1993), La prospective, pratiques et méthodes, Paris, Economica.

JACOB, R., P.-A. JULIEN et L. RAYMOND (1996), «L'organisation apprenante ou apprendre en réseau », IX ${ }^{\mathrm{e}}$ congrès de $\mathrm{l}^{\prime}$ Association internationale de psychologie du travail de langue française, Sherbrooke, 27-29 août.

Joly, M. (1993), Introduction à l'analyse de l'image, Paris, Nathan Université.

JULIEN, P.-A. (1989), «The entrepreneur and economic theory», International Small Business Journal, vol. 7, n 3, p. 29-38.

Julien, P.-A. (2002), «Pour une définition de la P.M.E.», dans P.-A. Julien (dir.), Les PME: Bilan et perspectives, $3^{\mathrm{e}}$ édition, Québec, Presses Inter Universitaires, p. $1-16$.

Julien, P.-A., M. CARrier, L. DÉSAulniers, D. LuC et Y. MARTineau (2000), « Les PME à forte croissance, comment gérer l'improvisation de façon cohérente», Actes du $V^{e}$ Colloque international francophone de la PME, Lille.

JULIEN, P.-A. et M. MARChESNAY (1992), «Des procédures aux processus stratégiques dans la PME », Piccola impresa, no 1, p. 13-41.

KAPLAN, R.S. (1998), «Innovative action research : creating new management theory and practice », Journal of Management Accounting Research, vol. 10, n 4, p. 89-118.

KoENIG, G. (1996), Management stratégique : paradoxes, interactions et apprentissages, Paris, Nathan.

LACROUX, F. (1999), «La modélisation dans le contrôle de gestion », dans Y. Dupuis (dir.), Faire de la recherche en contrôle de gestion?, Paris, Vuibert-Fnege, p. 21-29.

LANDRY, M. (1983), «Qu'est-ce qu'un problème ?», Cahiers de recherche de l'équipe en système d'information organisationnelle, Faculté des sciences de l'administration, Université Laval, Québec.

LE Moigne, J.-L. (1977), La théorie du système général, Paris, Presses universitaires de France.

Le Moigne, J.-L. (1990), La modélisation des systèmes complexes, Paris, Dunod.

LEFEBVRE, J.-F. (1982), L'analyse structurelle: méthodes et développements, Thèse de doctorat de $3^{\mathrm{e}}$ cycle, Paris IX Dauphine.

Revue internationale P.M.E., vol. 15, $\mathrm{n}^{\circ}$ 2, 2002 
LoPEZ-Monsalvo, C. (1998), Incertitude en pilotage de projets innovants : approche conceptuelle et contribution méthodologique, Thèse de doctorat, Nancy, INPLLRGSI.

LORINO, P. (1999), «Le sens giratoire et le chameau », dans Entre systémique et complexité, chemin faisant..., Paris, Presses universitaires de France, p. 147-156.

LUKKA, K. (1998), «Constructive approach to field research in management accounting », Cahiers de recherche, EIASM, Bruxelles.

Marchesnay, M. et C. CARrier (2002), «Le management stratégique », dans P.-A. Julien (dir.), Les PME: Bilan et perspectives, $3^{\mathrm{e}}$ édition, Québec, Presses Inter Universitaires.

MARCHINI, I. (1995), Il governo della piccola impresa, tome 2, Gènes, ASPI/INS-EDIT.

MARTEL, M. et M. OrAL (1995), «L'entreprise au cœur de la compétitivité », dans Les défis de la compétitivité, Montréal, Publi-relais.

Martinet, A.-C. (1993), «Stratégie et pensée complexe», Revue française de gestion, $\mathrm{n}^{\circ}$ 93, mars-avril-mai, p. 64-72.

Martinet, A.-C. (1996), Préface de B. Aliouat, Les stratégies de coopération industrielle, Paris, Economica.

Messeghem, K. et N. VARRAUT (1998), «Stratégie d'adoption d'une démarche qualité en PME», Revue internationale PME, vol. 11, $\mathrm{n}^{\circ} 1$, p. 101-122.

MiChEL, J. (1997), «L'Analyse de la valeur adaptée à la reconfiguration de petites et moyennes entreprises », La Valeur, no 69 , juillet, p. 17-20.

MORIN, E. (1990), Introduction à la pensée complexe, Paris, ESF.

Morin, E. et J.L. Le Moigne (1999), L’intelligence de la complexité, Paris, L'Harmattan.

NicOT, A.-M. (1997), «L'intervention de conseil », dans M.-J. Avenier (dir.), La stratégie «chemin faisant... », Paris, Economica, p. 219-238.

PIAGET, J. (1973), La construction du réel chez l'enfant, 5e édition, Neuchâtel, Delachaux et Niestlé.

Probst, G.J.B. et B.S.T. BüCHEL (1995), Organisationales Lernen, Gaber, traduction française, La pratique de l'entreprise apprenante, Paris, Éditions d'Organisation.

RoY, B. (1992), «Sciences de la décision ou science de l'aide à la décision?», Revue internationale de systémique, vol. 6, n 5, p. 497-529.

SCHLANGER, J. (1990), La situation cognitive, Paris, Méridiens.

SCHMITT, C. (1999), La dynamique de la valeur: contribution à la création de valeur en PME par la notion de désordre, Thèse de doctorat, Nancy.

SCHMITT, C. et M. BAYAD (2000), «Création de valeur et désordre en PME: vers le développement d'une recherche ingénierique » Actes $d u V^{e}$ Congrès international francophone sur la PME, Lille, 25-27 octobre 2000.

Revue internationale P.M.E., vol. 15, $\mathrm{n}^{\circ}$ 2, 2002 
SCHMITT, C. et J.P. GRANDHAYE (1999-2000), « Ordre et désordre en PME : contribution du visuel au développement organisationnel », Direction et Gestion, $\mathrm{n}^{\text {os }} 180-181$, p. $45-60$.

SERVANT, D. (1990), «Le rôle des images dans les sciences de gestion », CXV Congrès national des sociétés savantes, Avignon, L’image et la science, p. 387-409.

Simon, H.A. (1991), The Sciences of the Artificial, Cambridge, Massachusetts Institute of Technology, traduction française Sciences des systèmes, sciences de l'artificiel, Paris, Dunod.

TARONDEAU, J.C. (1998), Le management des savoirs, Paris, Presses universitaires de France, coll. «Que sais-je?».

TORRÈs, O. (1999), Les P.M.E., Paris, Flammarion-Dominos.

Verstraete, T. (1997), «Cartographie cognitive et accompagnement du créateur d'entreprise », Revue internationale PME, vol. 10, n 1, p. 43-72.

Watzlawick, P. (1988), «Effet ou cause?», dans P. Watzlawick (dir.), L'invention de la réalité : contribution au constructivisme, Paris, Seuil, p. 73-78.

Watzlawick P., J. Weakland et R. Fisch (1974), Change. Principles of Problem Formation and Problem Resolution, New York, W.W. Norton.

Weinberg, A. (1995), «Les jeux de l'ordre et du désordre », Sciences humaines, nº 47, février, p. 16-18.

YATCHINOVSKY, A. (1999), L'approche systémique, pour gérer l'incertitude et la complexité, Paris, ESF. 